\title{
Informações Editoriais 2017
}

As informações abaixo são referentes aos fascículos 97, 98 e 99 do volume 33, publicados no ano de 2017.

\section{Demanda de Publicação}

\begin{tabular}{lc}
\hline Trabalhos & $\mathbf{2 0 1 7}$ \\
\hline Artigos submetidos & 221 \\
\hline Artigos rejeitados & 153 \\
\hline Artigos aceitos & 07
\end{tabular}

\section{Origem institucional dos autores}

\begin{tabular}{lccccc}
\hline Fascículos & USCS & $\begin{array}{c}\text { Outras } \\
\text { Instituições de } \\
\text { São Paulo }\end{array}$ & $\begin{array}{c}\text { Outras } \\
\text { instituições } \\
\text { nacionais }\end{array}$ & $\begin{array}{c}\text { Outras } \\
\text { instituições } \\
\text { internacionais }\end{array}$ & Total \\
\hline 97 & 3 & 3 & 22 & 0 & 31 \\
\hline 98 & 6 & 8 & 17 & 0 & 31 \\
\hline 99 & 9 & 16 & 66 & 0 & 91 \\
\hline Total & $\mathbf{9}$ & $\mathbf{1 6}$ & $\mathbf{6 6}$ & $\mathbf{0}$ & $\mathbf{9 1}$ \\
\hline & $\mathbf{9 , 8 9 \%}$ & $\mathbf{1 7 , 5 8 \%}$ & $\mathbf{7 2 , 5 3 \%}$ & $\mathbf{0 \%}$ & $\mathbf{1 0 0 \%}$ \\
\hline
\end{tabular}

\section{Origem geográfica dos autores}

\begin{tabular}{|c|c|c|c|c|c|}
\hline Origem geográfica & $\mathbf{N}^{\circ}$ de autores & $\%$ & Origem geográfica & $N^{\circ}$ de autores & $\%$ \\
\hline São Paulo & 25 & 27,48 & Piauí & 01 & 1,09 \\
\hline Minas Gerais & 14 & 15,39 & Rio de Janeiro & 01 & 1,09 \\
\hline Santa Catarina & 03 & 3,29 & Mato Grosso do Sul & 03 & 3,29 \\
\hline Rio Grande do Sul & 07 & 7,70 & Alagoas & 02 & 2,19 \\
\hline Paraná & 01 & 1,09 & Goiás & 03 & 3,29 \\
\hline Ceará & 05 & 5,49 & Sergipe & 01 & 1,09 \\
\hline Bahia & 22 & 24,18 & Espanha & 00 & 0,00 \\
\hline Brasília & 03 & 3,29 & Total & 91 & 100 \\
\hline
\end{tabular}

Número de artigos e autores por área temática

\begin{tabular}{lcccc}
\hline Área temática & Artigos & $\%$ & Autores & $\%$ \\
\hline Administração da Informação & 01 & 6,66 & 03 & 3,29 \\
\hline Empreendedorismo & 03 & 10,00 & 08 & 8,79 \\
\hline Ensino e Pesquisa em Administração e Contabilidade & 01 & 3,33 & 05 & 5,49 \\
\hline Estratégia em Organizações e Redes de Negócios & 06 & 20,00 & 17 & 18,68 \\
\hline Estudos Organizacionais & 04 & 13,33 & 13 & 14,28 \\
\hline Finanças & 02 & 6,66 & 16 & 6,59 \\
\hline Políticas Públicas e Desenvolvimento Regional & 08 & 26,66 & 28 & 27,48 \\
\hline Gestão de Pessoas e Relações de trabalho & 02 & 6,66 & 04 & 4,40 \\
\hline Sustentabilidade & 02 & 6,66 & 07 & 7,70 \\
\hline Marketing & 01 & 3,33 & 03 & 3,29 \\
\hline Total & $\mathbf{3 0}$ & $\mathbf{1 0 0 , 0}$ & $\mathbf{9 1}$ & $\mathbf{1 0 0}$ \\
\hline
\end{tabular}




\section{Nominata de pareceristas ad hoc}

Em nome da Universidade Municipal de São Caetano do Sul - USCS, formalizam-se agradecimentos aos pareceristas aqui relacionados, pela dedicação na avaliação dos artigos submetidos, o que se refletiu na celeridade das entregas e na qualidade das análises.

\begin{tabular}{|c|c|}
\hline Nome & Instituição de Ensino Superior \\
\hline Adonai Teles de Siqueira Sousa & Universidade Federal Fluminense \\
\hline Adriana Thomazotti Claro & Universidade Nove de Julho - UNINOVE \\
\hline Alba Zucco & Universidade Nove de Julho - UNINOVE \\
\hline Alexandre de Pádua Carrieri & Universidade Federal de Minas Gerais \\
\hline Alexandre Ripamonti & Universidade de São Paulo - FEA \\
\hline Almir Martins Vieira & Universidade Metodista de São Paulo \\
\hline Ana Clara Cândido & $\begin{array}{l}\text { Centro Interdisciplinar de Ciências Sociais - Universidade Nova de Lisboa, CICS.NOVA, } \\
\text { Portugal }\end{array}$ \\
\hline Ana Elizabeth Neirão Reymão & Universidade Federal do Pará - UFPA \\
\hline Ana Lucia Medeiros & Universidade Paulista - UNIP-Ribeirão Preto \\
\hline Ana Maria Auler Matheus Peres & Faculdade Arthur Sá Earp Neto - FASE \\
\hline Ana Maria auler Matheus Peres & Universidade Estadual do Rio de Janeiro - Instituto de Medicina Social \\
\hline Anapatrícia Morales Vilha & Universidade Federal do ABC - UFABC \\
\hline Antonio Carlos Zambon & Universidade Estadual de campinas - UNICAMP - Fac. de Tecnologia \\
\hline Antonio Marcos Vargas de Oliveira & Universidade Cruzeiro do Sul \\
\hline Ariel Levy & Universidade Federal Fluminense - UFF \\
\hline Benedito Cabral de Medeiros Filho & Fundação Getulio Vargas - FGV \\
\hline Carlos Alberto Di Agustini & Fundação Getúlio Vargas - FGV e Universidade Municipal de São Caetano do Sul - USCS \\
\hline Celso Machado Júnior & Complexo Educacional FMU \\
\hline Cibele Roberta Sugahara & Pontifícia Universidade Católica de Campinas - PUC-Campinas \\
\hline Claudio Zancan & Universidade Federal de Alagoas \\
\hline Daniel Knebel Baggio & Universidade Regional do Noroeste do Estado do Rio Grande do Sul \\
\hline Dario de Oliveira Lima Filho & Universidade Federal de Mato Grosso do Sul \\
\hline Darticléia Almeida Sampaio da Rocha Soares & Universidade Municipal de São Caetano do Sul - USCS \\
\hline Denis Donaire & Universidade Municipal de São Caetano do Sul - USCS \\
\hline Dirceu Silva & Uninove e Unicamp \\
\hline Edenis Cesar de Oliveira & Universidade Federal de São Carlos - UFSCar \\
\hline Edgar Reyes Junior & Universidade do Vale do Rio dos Sinos - UNISINOS \\
\hline Edilson dos Santos Silva & Faculdade Estácio de Alagoas \\
\hline Edmir Parada VasquesPrado & Universidade de São Paulo \\
\hline Eliane Maria Giavina Bianchi & Fundação Getúlio Vargas e HSM Educação \\
\hline Elmo Tambosi Filho & Universidade Metodista de São Paulo \\
\hline Elvis Silveira Martins & Universidade Federal de Pelotas - UFPel \\
\hline Ericson Saint Clair & Universidade Federal Fluminense - UFF \\
\hline Fabricia Silva da Rosa & Universidade Federal de Santa Catarina \\
\hline Fernando Coutinho Garcia & Faculdade Novos Horizontes \\
\hline Genaro Aguilar Gutiérrez & IPN México \\
\hline Gino Giacomini Filho & Universidade de São Paulo - USP \\
\hline Helio Rubens Jacintho Pereira Junior & $\begin{array}{l}\text { Escola Superior de Gestão de Negócios, Escola Superior de Adm. Marketing e } \\
\text { Comunicação de Sorocaba }\end{array}$ \\
\hline Henrique Cesar Melo Ribeiro & Universidade Nove de Julho - Uninove \\
\hline Hilka Pelizza Vier Machado & Universitário de Maringá - UniCesumar \\
\hline Izabel Cristina Petraglia & Centro Universitário das Faculdades Metropolitanas Unidas \\
\hline Jamerson ViegasQueiroz & Universidade Federal do Rio Grande do Norte \\
\hline
\end{tabular}




\section{Gestão \& Regionalidade}

\begin{tabular}{|c|c|}
\hline Nome & Instituição de Ensino Superior \\
\hline Janis Elisa Ruppenthal & Universidade Federal de Santa Maria \\
\hline Jarbas Thaunahy Santos de Almeida & Universidade Municipal de São Caetano do Sul - USCS \\
\hline José Carlos de Souza Lima & Universidade Cruzeiro do Sul \\
\hline José Carlos Silva Filho & Unidade de Ensino Superior Dom Bosco \\
\hline José Carlos Thomaz & Universidade Mackenzie \\
\hline José Eduardo Rodrigues de Sousa & Pontifícia Universidade Católica de Campinas \\
\hline Josilmar Cordenonssi Cia & Universidade Presbiteriana Mackenzie \\
\hline Julio Carneiro da Cunha & Universidade Nove de Julho - UNINOVE \\
\hline Luci Mendes de Mello Bonini & Universidade de Mogi das Cruzes \\
\hline Luciene Eberle & Universidade de Caxias do Sul \\
\hline Márcia Freire Oliveira & Universidade Federal de Uberlândia \\
\hline Marcia Horowitz Vieira & Pontifícia Universidade Católica do Rio Grande do Sul - UCRS \\
\hline Márcia Freire de Oliveira & Universidade Federal de Uberlândia \\
\hline Márcio Shoiti Kuniyoshi & Universidade Metodista de São Paulo \\
\hline Marco Aurélio Sanches Fittipaldi & Universidade Guarulhos \\
\hline Marcos Vinicius Pó & Universidade Federal do $A B C$ - UFABC \\
\hline Marcos Antonio Maia de Oliveira & Centro Estadual de Educação Tecnológica Paula Souz \\
\hline Marcos Georges & Pontifícia da Universidade Católica - PUC-Campinas \\
\hline Marcos Ricardo Rosa Georges & Pontifícia da Universidade Católica - PUC-Campinas \\
\hline Marcos Roberto Pinto & Universidade Federal do Rio de Janeiro - UFRJ. \\
\hline Marcus Vinicius de Oliveira Brasil & Universidade Federal do Cariri - UFCA \\
\hline Maria das Graças Gonçalves Vieira Guerra & Universidade Federal de Pernambuco - UFPE \\
\hline Maria Eugenia Porém & Universidade Estadual Paulista - UNESP \\
\hline Michel Mott Machado & Universidade de São Paulo - USP \\
\hline Moisés Ferreira Cunha & Universidade Federal de Goiás \\
\hline Monica Franchi Carniello & Universidade Taubaté - UNITAU \\
\hline Nadia Kassouf Pizzinato & Universidade Nove de Julho - Uninove \\
\hline Patricia Campeão & Universidade Federal de Mato Grosso do Sul \\
\hline Paulo Ramirez & Instituto Federal de Tecnologia de São Paulo - IFSP \\
\hline Priscila Casari & Universidade Federal de Goiás - UFG \\
\hline Rafael Lübeck & Universidade de Caxias do Sul - UCS \\
\hline Rafael Mendes Lübeck & Universidade de Caxias do Sul - UCS \\
\hline Rafael Ricardo Jacomossi & Centro Universitário da FEI \\
\hline Renato de Castro Garcia & Universidade de São Paulo - USP \\
\hline Reynaldo Cavalheiro Marcondes & Universidade Presbiteriana Mackenzie \\
\hline Ricardo Rossetti & Universidade Metodista de São Paulo \\
\hline Richard Medeiros Araújo & Centro Universitário FACEX \\
\hline Robéria Rodrigues Lopes & Universidade Federal do Ceará \\
\hline Rosana Tavares & Fundação Instituto de Administração - FIA \\
\hline Rosângela Sarmento Silva & Universidade Federal de Sergipe - UFS \\
\hline Sandra Mara Schiavi Bánkuti & Universidade Estadual de Maringá - PR \\
\hline Senichiro Koshio & Universidade Presbiteriana Mackenzie \\
\hline Sérgio Luiz do Amaral Moretti & Universidade Anhembi Morumbi \\
\hline Silvia Helena de Camargo Ramos Valadão de Camargo & Centro Universitário Moura Lacerda de Ribeirão Preto e Anhanguera Educacional \\
\hline Sirlei Tereza Pitteri Vieira & Universidade Paulista - UNIP \\
\hline Sulivan Desirée Fischer & Universidade do Estado de Santa Catarina - ESAG/UDESC \\
\hline Suzete Antonieta Lizote & Universidade do Vale de Itajaí - Univali \\
\hline Vanessa Regina Cenedezi Zanarotti & Universidade Paulista - UNIP-Ribeirão Preto \\
\hline
\end{tabular}




\section{Índice por Área Temática 2017}

\section{ADMINISTRAÇÃO DA INFORMAÇÃO}

A TECNOLOGIA DA INFORMAÇÃO E OS MOVIMENTOS SOCIAIS: UM ESTUDO DO MOVIMENTO PASSE LIVRE

Gustavo Hermínio Salati Marcondes de Moraes, Alexandre

Cappellozza, Fernando de Souza Meirelles

Gestão \& Regionalidade - Vol. 33 - № 97, pp. 137-153, jan-abr/2017

doi: 10.13037/gr.vol33n97.3983

\section{EMPREENDEDORISMO}

\section{CARACTERÍSTICAS EMPREENDEDORAS DO} PRODUTOR RURAL CAPIXABA

Juliana Binow Ferreira, Sarah Venturim Lasso, Emerson Mainardes Gestão \& Regionalidade - Vol. 33 - No 99, pp. 74-90, set-dez/2017 doi: 10.13037/gr.vol33n99.2943

RELAÇÃO ENTRE INTRAEMPREENDEDORISMO E INOVAÇÃO: UM ESTUDO EM EMPRESAS PARTICIPANTES DO APL DE TI DO SUDOESTE DO PARANÁ

Helem Baldisera, Gilberto Francisco Ceretta, Dálcio Roberto dos Reis Gestão \& Regionalidade - Vol. 33 - No 97, pp. 100-117, jan-abr/2017 doi: $10.13037 /$ gr.vol33n97.3740

A INFLUÊNCIA DAS ARENAS ESPORTIVAS NA CRIAÇÃO E SOBREVIVÊNCIA DE MICRO E PEQUENOS NEGÓCIOS

Silvia Simoni Orlando, Vania Maria Nassif

Gestão \& Regionalidade - Vol. 33 - № 98, pp. 20-35, maio-ago/2017 doi: $10.13037 /$ gr.vol33n98.3748

\section{ENSINO E PESQUISA EM ADMINISTRAÇÃO E CONTABILIDADE}

ESTEREÓTIPO DA IMAGEM DE PAÍS: ANÁLISE DA PERCEPÇÃO DOS ESTRANGEIROS EM RELAÇÃO AO BRASIL Ana Paula Perlin, Maria Carolina Fagundes, Flávia luciane Scherer, Clandia Maffini Gomes, Jordana Marques Kneipp Gestão \& Regionalidade - Vol. 33 - № 98, pp. 53-66, maio-ago/2017 doi: 10.13037/gr.vol33n98.4039

\section{ESTRATÉGIAS ORGANIZAÇÕES E REDES DE NEGÓCIOS}

CLUSTERS COMERCIAIS: ESTUDO SOBRE UMA AGLOMERAÇÃO DE LOJAS TÊXTEIS EM SÃO PAULO

Esdras Silva Costa, Denis Donaire, Marcos Antonio Gaspar Gestão \& Regionalidade - Vol. 33 - No 98, pp. 04-19, maio-ago/2017 doi: $10.13037 /$ gr.vol33n98.3336

PRESSUPOSTOS DO DESEMPENHO: UM ESTUDO DA AMBIDESTRIA, EXPLORAÇÃO E EXPLOTAÇÃO EM UMA REDE DE FARMÁCIAS DO RS

Juliana de Lima Marques, Elvis Silveira-Martins

Gestão \& Regionalidade - Vol. 33 - № 97, pp. 118-136, jan-abr/2017 doi: 10.13037/gr.vol33n97.3831

COMO AS AÇÕES AUTÔNOMAS INFLUENCIAM O PROCESSO DE FORMAÇÃO DA ESTRATÉGIA NA
GESTÃO DO CURSO EAD DE ADMINISTRAÇÃO PÚBLICA DA UNIVERSIDADE FEDERAL DE SANTA CATARINA

Guilherme Rizzatti, Rosalia Aldraci Barbosa Lavarda Gestão \& Regionalidade - Vol. 33 - № 98, pp. 67-82, maio-ago/2017 doi: 10.13037/gr.vol33n98.3850

ANÁLISE DE REDES SOCIAIS NO ARRANJO PRODUTIVO LOCAL DOS RAMOS TÊXTIL E DE CONFECÇÕES DA REGIÃO DA GRANDE SÃO PAULO A PARTIR DE UMA VISÃO DE GOVERNANÇA

Milton Carlos Farina, Alessandra Preto Bitante, luciane Ribeiro Dias Pinheiro, Lidiane Campos Britto

Gestão \& Regionalidade - Vol. 33 - № 98, pp. 36-52, maio-ago/2017 doi: 10.13037/gr.vol33n98.3791

ANÁLISE DOS FATORES CONDICIONANTES DA GÊNESE DE CLUSTERS DE EMPRESAS

Simone Ruchdi Barakat, Taiguara Langrafe, Maria Laura Ferranty Maclennan, João Maurício Gama Boaventura

Gestão \& Regionalidade - Vol. 33 - № 98, pp. 135-152, maio-ago/2017 doi: 10.13037/gr.vol33n98.3994

RELAÇÃO ENTRE MONITORAMENTO E ORIENTAÇÃO ESTRATÉGICA: UM ESTUDO DA PERCEPÇÃO DE PROFISSIONAIS DA INTELIGÊNCIA COMPETITIVA Maria Cristina Pereira Pimentel, Fernando Carvalho de Almeida Gestão \& Regionalidade - Vol. 33 - Nº 99, pp. 128-145, set-dez/2017 doi: 10.13037/gr.vol33n99.3325

\section{ESTUDOS ORGANIZACIONAIS}

VÍNCULOS ORGANIZACIONAIS: O CASO DE DOCENTES DE UMA INSTITUIÇÃO PRIVADA DE ENSINO SUPERIOR LOCALIZADA EM BELO HORIZONTE, MINAS GERAIS Ricardo Bastos Rodrigues, luiz Carlos Honório Gestão \& Regionalidade - Vol. 33 - № 98, pp. 98-117, maio-ago/2017 doi: $10.13037 /$ gr.vol33n98.3956

RESPONSABILIDADE SOCIAL CORPORATIVA E O DESEMPENHO FINANCEIRO NO SETOR DE ENERGIA ELÉTRICA: UM ESTUDO COM MODELO DE DADOS EM PAINÉIS

Flávio Ribeiro, Thays Aparecida Alves, Marines Taffarel, Gelson MENON

Gestão \& Regionalidade - Vol. 33 - No99,pp.39-54, set-dez/2017 doi: 10.13037/gr.vol33n99.3987

VALORES ORGANIZACIONAIS: PREDITORES DO BEMESTAR NO TRABALHO?

Liane Terezinha Braga Rissi, Tais de Andrade, Vania de Fátima Barros Estivalete, Jefferson Menezes de Oliveira, Vívian Flores Costa Gestão \& Regionalidade - Vol. 33 - N99, pp.170-187, set-dez/2017 doi: $10.13037 /$ gr.vol33n99.4091

GRAU DE MATURIDADE NA GESTÃO DAS EMPRESAS DE CONFECÇÃO EM SANTA CRUZ DO CAPIBARIBE (PE)

Stêvenis Moacir Moura da Fonseca, Andréa Pereira da Silva Gestão \& Regionalidade - Vol. 33 - No 97, 35-82, jan-abr/2017 doi: $10.13037 /$ gr.vol33n97.3573 


\section{FINANÇAS}

RISCO SISTÊMICO E EFEITO CONTÁGIO À LUZ DO MÉTODO COVAR: UMA ANÁLISE COM AS AÇÕES MAIS LÍQUIDAS LISTADAS NA BM\&FBOVESPA

Ana Paola Fernandes, Márcio André Veras Machado, Paulo

Roberto Nóbrega Cavalcante

Gestão \& Regionalidade - Vol. 33 - No99 - set-dez/2017

doi: 10.13037/gr.vol33n99.4165

A RELAÇÃO ENTRE INTENSIDADE TECNOLÓGICA E GRAU DE INTANGIBILIDADE NO DESEMPENHO ECONÔMICOFINANCEIRO DA INDÚSTRIA BRASILEIRA

Guilherme Afrânio Machado, luciana Carvalho, Fernanda Maciel PEIXOTO

Gestão \& Regionalidade - Vol. 33 - No99, pp.1-18, set-dez/2017

doi: $10.13037 /$ gr.vol33n99.4167

\section{GESTÃO DE PESSOAS E RELAÇÕES DE TRABALHO}

A SÍNDROME DE BURNOUT ESTÁ ASSOCIADA AO

TRABALHO DOS AUDITORES INTERNOS?

Daniele Cristina Bernd, Ilse Maria Beuren

Gestão \& Regionalidade - Vol. 33 - N99, pp.146-169, set-dez/2017 doi: 10.13037/gr.vol33n99.3408

GESTÃO DE RECURSOS HUMANOS À LUZ DA RESPONSABILIDADE SOCIAL EMPRESARIAL: UM ESTUDO NO SETOR SUCROENERGÉTICO EM GOIÁS

José Elenilson Cruz, Eliane Moreira Sá de Souza

Gestão \& Regionalidade - Vol. 33 - No 97, pp.23-47, jan-abr/2017

doi: 10.13037/gr.vol33n97.2909

\section{POLÍTICAS PÚBLICAS E DESENVOLVIMENTO REGIONAL}

DESENVOLVIMENTO REGIONAL SOB A ÓTICA DO RECONHECIMENTO DA INDICAÇÃO GEOGRÁFICA: O CASE DO VALE DOS VINHEDOS, A PARTIR DA PERCEPÇÃO DOS ATORES SOCIAIS REGIONAL

Dieter Rugard Siedenberg, Aleteia Hummes Thaines, Daniel Knebel BAGGIO

Gestão \& Regionalidade - Vol. 33 - N99, pp.04-20 - set-dez/2017 doi: 10.13037 /gr.vol33n99.2771

SOBRE INCENTIVOS FISCAIS MUNICIPAIS E O DESENVOLVIMENTO LOCAL DE MONTES CLAROS (MG): DILEMAS E PERCEPÇÕES SOBRE "CIDADES ATRATIVAS"

Felipe Fróes Couto, Ivan Beck Ckagnazaroff

Gestão \& Regionalidade - Vol. 33 - No 98, pp.83-97, maio-ago/2017 doi: $10.13037 /$ gr.vol33n98.3853

PRODUÇÃO DE INFORMAÇÃO PARA POLÍTICA HABITACIONAL NO CONTEXTO DOS PEQUENOS MUNICÍPIOS: ALTERNATIVA DE UTILIZAÇÃO DO SISTEMA DE INFORMAÇÃO DA ATENÇÃO BÁSICA (SIAB)

Rosana Denaldi, Maria de lourdes Fonseca, Ana Gabriela Akaishi Gestão \& Regionalidade - Vol. 33 - N999, pp.55-73, set-dez/2017 doi: $10.13037 /$ gr.vol33n99.3806

CIDADES MÉDIAS DO NORDESTE: BREVES CONSIDERAÇÕES ACERCA DOS DINAMISMOS E DESAFIOS NO PÓS-1990
William Eufrásio Nunes Pereira, Ana Cristina Santos Morais, Aline Alves Oliveira

Gestão \& Regionalidade - Vol. 33 - No 97, pp.5-22, jan-abr/2017 doi: 10.13037/gr.vol33n97.2896

IMPLICAÇÕES DO ESFORÇO DE ARRECADAÇÃO NO DESEMPENHO SOCIOECONÔMICO DOS MUNICÍPIOS DE MINAS GERAIS

Michelle Aparecida Vieira, luiz Antonio Abrantes, Marco Aurélio Marques Ferreira, Jéssika do Vale Silva

Gestão \& Regionalidade - Vol. 33 - N 99, pp.21-38, set-dez/2017 doi: $10.13037 /$ gr.vol33n99.3981

DESENVOLVIMENTO RURAL DOS MUNICÍPIOS DE MINAS GERAIS: FATORES DETERMINANTES E HIERARQUIZAÇÃO

luana Ferreira dos Santos, Marco Aurélio Marques Ferreira, Rafael Júnior dos Santos Figueiredo Salgado

Gestão \& Regionalidade - Vol. 33 - N 97, pp.83-99, jan-abr/2017 doi: 10.13037/gr.vol33n97.3640

GESTÃO DOS RECURSOS DO SISTEMA ÚNICO DE SAÚDE NA BAHIA: UMA ANÁLISE CONSIDERANDO A INFLUÊNCIA DOS CICLOS ELEITORAIS NO ÍNDICE DE EFICIÊNCIA MUNICIPAL

Thaís lima Fraga, Paulo Ramos, Ricardo André da Costa, Adriano Provezano Gomes

Gestão \& Regionalidade - Vol. 33 - № 97, pp.154-169, jan-abr/2017 doi: $10.13037 /$ gr.vol33n97.4023

(RE)SIGNIFICANDO O ESPAÇO COMO CAPITAL: CONTRIBUIÇÕES DE ESTUDO JUNTO À RUA SANTA JULIANA, SETE LAGOAS, MG

Anderson de Souza Sant'anna, Daniela Martins Diniz, Fátima BAYMA DE OLIVEIRA

Gestão \& Regionalidade - Vol. 33 - № 98, pp.118-135, maio-ago/2017 doi: $10.13037 /$ gr.vol33n98.4079

\section{SUSTENTABILIDADE}

TENDÊNCIAS DE PESQUISA EM GESTÃO DA CADEIA DE SUPRIMENTOS VERDE

Mayra Cabrera Costa, Fabiane de Deus Teixeira, Márcio lopes

Pimenta, luciana Oranges Cezarino

Gestão \& Regionalidade - Vol. 33 - № 98, pp.153-166, maio-ago/2017 doi: 10.13037/gr.vol33n98.4007

GESTÃO AMBIENTAL E GESTÃO DOS RECURSOS HÍDRICOS NO CONTEXTO DO USO E OCUPAÇÃO DO SOLO NOS MUNICÍPIOS

Ruan Carlos de Mesquita Oliveira, Patrícia Verônica Pinheiro Sales Lima, Rennaly Patrício Sousa

Gestão \& Regionalidade - Vol. 33 - № 97,pp.48-64, jan-abr/2017 doi: $10.13037 / g r . v o l 33 n 97.3101$

\section{MARKETING}

O MARKETING INTERNO COMO ELEMENTO BÁSICO AO DESENVOLVIMENTO DE CIDADES E LOCALIDADES

Renato Henrique da Luz, Roseneide Maria de Lima, Silvio Augusto MINCIOTTI

Gestão \& Regionalidade - Vol. 33 - Nº 97, pp.170-181, jan-abr/2017 doi: $10.13037 /$ gr.vol33n97.4095 